\title{
Why paediatricians should be aware off Adverse Childhood Experiences? Let's stick together!
}

\author{
Vanja Saftić, Child and Youth Protection Center Zagreb, Croatia
}

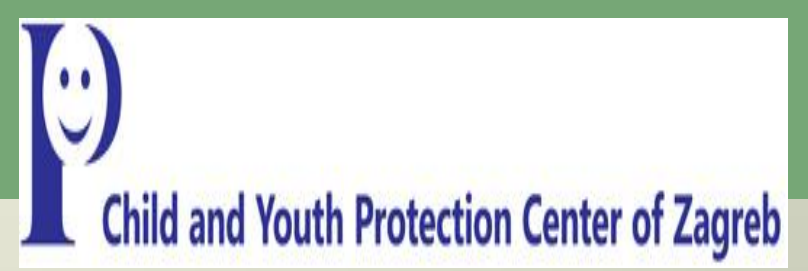

Objective

The effects of negative environmental factors in childhood result in neuro-biological changes, which consequently may cause changes to the cytoarchitecture of the brain, and reduce or change their capacities and potentials. Exposed to such powerful, negative environment, lead to hyperactivation of the neurological and endocrine system. This state is known as a toxic stress. It causes short-term consequences, predominantly with neuro-developmental disorders, cognitive and psychosocial decline. Symptoms may frequently imitate ADHD. The EEG, in a case of nmay wa significant number of nonspecific changes, and in especially brutal forms, epileptic brain activity. The aim of this study was to estimate preliminary results of a multi-disciplinary clinical assessment of patient came to Child and Youth protection Center, Zagreb, Croatia, because of adverse childhood experiences, acording ACEs (Adverse Childhood Experience score) $\geq 4$.

\section{Methods}

From 2015 - 2017 in Child and Youth Protection Center Zagreb we prospectively observed 954 children who experienced abuse and/or neglect, with ACES (Adverse Childhood Experiences Score) $\geq 4$. We used established questionnaire from the Centers for Disease Control and Prevention / short ACE tool with questions measuring childhood exposure to adverse experiences. Our multidisciplinary team (Pediatric neurologist, Social worker, Psychologist, Psychiatrist, Speech therapist and special need expert) evalutaed their tests. Mean age for our patients was 6,9 year. Every child underwent Touwen Neurological Examination. In this follow-up study we were looking for presence of minor neurological dysfunctions (gross motor function, mild dysfunction in posture, reflexes, coordination, fine manipulative ability, fine motor disfunction, dyskinesia and excessive associated movements). For those with ACES $\geq 4$, and neurodevelopmental delay, EEG was indicated We measured height and weight and compared with percentile values, for every participant. We also evaluated social interactions and behaviour risk.

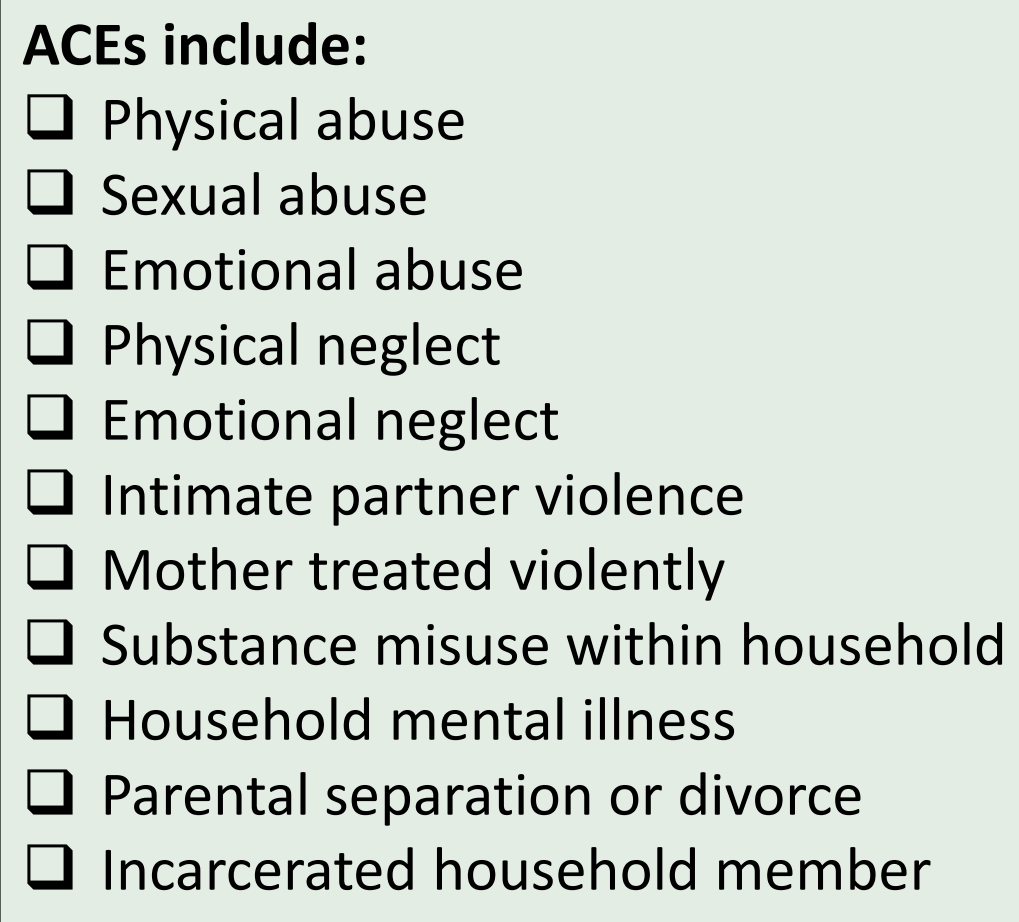

Results

Neglect, physical and emotional abuse and high conflict divorce are the most important adverse experiences resulting in deviations in neurological development. Impulsive behaviour, and other form of ADHD "like" variants are significantly more frequent in children with history of some kind of abuse or neglect.Over $70 \%$ of patients with ACES $\geq 4$ showed dysrhytmic or other form of nonspecific changes in EEG. Only $7 \%$ of them with experience of fetal abuse and/or neglect (Fetal alcohol syndrome, Neonatal abstinence syndrome) didn't have any neurodevelopment problems. Moreover, $19 \%$ of them, showed cognitive impairment, $33 \%$ had speech problems, and over $60 \%$ had learning problems.Impulsive behaviour, and other form of ADHD "like" variants are significantly more frequent (over $50 \%$ ) in children with history of some kind of abuse or neglect. Child faced with toxic stress are significantly more obese and overweight than their peers.
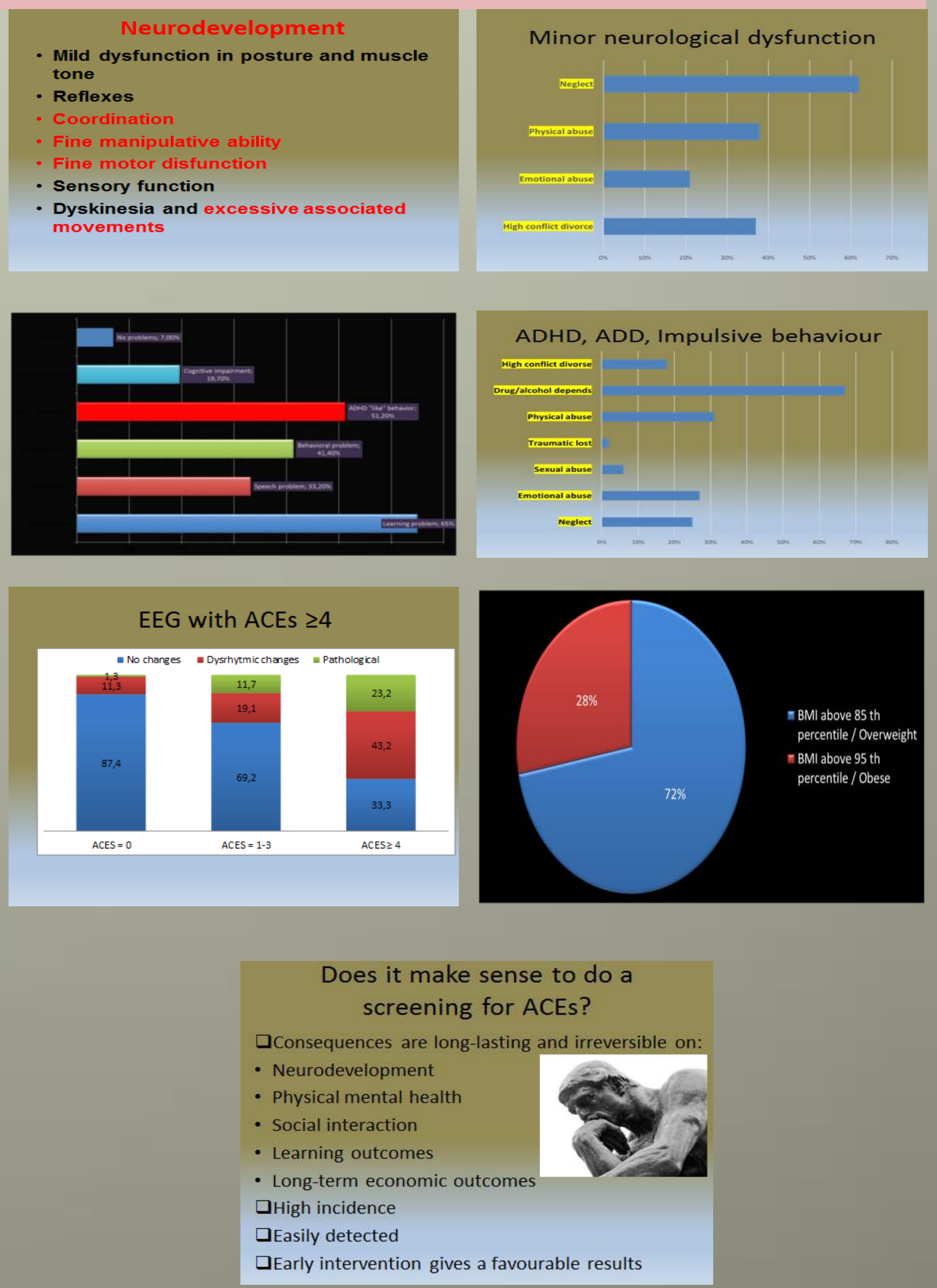

Conclusion

Early detection of adverse childhood experiences help to start with early interventions in order to prevent long term consequences of toxic stress. 